\title{
Development of a cadmium telluride pixel detector for astrophysical applications
}

Hiromasa Miyasaka, Fiona A. Harrison, Walter R. Cook, Peter H. Mao, Vikram R. Rana, et al.

Hiromasa Miyasaka, Fiona A. Harrison, Walter R. Cook, Peter H. Mao, Vikram R. Rana, Shin-nosuke Ishikawa, Masayoshi Ushio, Hiroyuki Aono, Shin Watanabe, Goro Sato, Motohide Kokubun, Tadayuki Takahashi, "Development of a cadmium telluride pixel detector for astrophysical applications," Proc. SPIE 7435, UV, X-Ray, and Gamma-Ray Space Instrumentation for Astronomy XVI, 74350Q (21 August 2009); doi: 10.1117/12.825711

EDent: SPIE Optical Engineering + Applications, 2009, San Diego, California, United States 


\title{
Development of a Cadmium Telluride pixel detector for astrophysical applications
}

\author{
Hiromasa Miyasaka ${ }^{a}$, Fiona A. Harrison ${ }^{a}$, Walter R. Cook $^{a}$, Peter H. Mao ${ }^{a}$, Vikram R. Rana ${ }^{a}$,

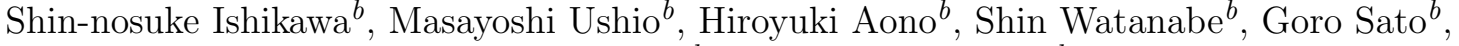 \\ Motohide Kokubun $^{b}$, Tadayuki Takahashi ${ }^{b}$ \\ ${ }^{a}$ Space Radiation Laboratory, California Institute of Technology, Pasadena, CA 91125, USA; \\ ${ }^{b}$ Institute of Space and Astronautical Science (ISAS/JAXA), Sagamihara, Kanagawa \\ 22908510, Japan
}

\begin{abstract}
We are developing imaging Cadmium Telluride (CdTe) pixel detectors optimized for astrophysical hard X-ray applications. Our hybrid detector consist of a CdTe crystal $1 \mathrm{~mm}$ thick and $2 \mathrm{~cm} \times 2 \mathrm{~cm}$ in area with segmented anode contacts directly bonded to a custom low-noise application specific integrated circuit (ASIC). The CdTe sensor, fabricated by ACRORAD (Okinawa, Japan), has Schottky blocking contacts on a 605 micron pitch in a $32 \times 32$ array, providing low leakage current and enabling readout of the anode side. The detector is bonded using epoxy-gold stud interconnects to a custom low noise, low power ASIC circuit developed by Caltech's Space Radiation Laboratory. We have achieved very good energy resolution over a wide energy range $(0.62 \mathrm{keV}$ FWHM @ 60keV, 10.8keV FWHM @ 662keV). We observe polarization effects at room temperature, but they are suppressed if we operate the detector at or below $0^{\circ} \mathrm{C}$ degree. These detectors have potential application for future missions such as the International X-ray Observatory (IXO).
\end{abstract}

Keywords: hard X-ray astrophysics, CdTe, anode pixelated detector, ASIC, International X-ray Observatory

\section{INTRODUCTION}

The next generation astrophysical observations with hard X-ray telescope will require low-power, compact detectors with good energy resolution and two-dimensional position resolution. For these reasons, a number of hard X-ray astrophysics missions currently under study or development plan to employ Cadmium Telluride (CdTe) or Cadmium Zinc Telluride (CdZnTe) detectors. Relative to the alkali halide scintillators or germanium detectors employed by previous-generation astrophysics experiments, CdTe offers the ability to implement position sensitive focal planes in compact geometries with reasonably good spectral resolution.

For decades, Caltech has been developing pixel detectors using Cadmium Zinc Telluride (CdZnTe) that meet the performance requirements for space base hard X-ray telescopes demanding good spectral and spatial resolution with very low power; in the range 50 to $100 \mu \mathrm{W}$ per pixel. ${ }^{1}$ The $\mathrm{ASIC}^{2}$ was originally developed for the High Energy Focusing Telescope (HEFT) balloon experiment, ${ }^{3}$ and has been improved subsequently for homeland security applications and, most recently, for the Nuclear Spectroscopic Telescope Array (NuSTAR) space mission. ${ }^{4}$

In order to improve hard X-ray imaging detection with CdTe, we have been developing pixel detectors in a collaboration between Caltech and ISAS. It is well known that Schottky-contact CdTe sensors $(\mathrm{In} / \mathrm{CdTe} / \mathrm{Pt})$ achieve lower leakage current and higher energy resolution than ohmic-contact $\mathrm{CdTe}$ sensors $(\mathrm{Pt} / \mathrm{CdTe} / \mathrm{Pt})$. Indium (In) electrodes form an anode side of Schottky barrier but are difficult to segment into pixels. In our previous work, we used ohmic-contact pixelated CdTe sensors hybridized with Caltech ASICs designed for HEFT balloon experiment. This combination of an ohmic-contact CdTe sensor and the HEFT ASIC, resulted in a good energy resolution and good pixel uniformity under cool conditions $\left(-20 \sim-50^{\circ} \mathrm{C}\right) .^{5}$

Further author information: (Send correspondence to H.M.)

H.M.: E-mail: miyasaka@srl.caltech.edu, Telephone: 16263952189

UV, X-Ray, and Gamma-Ray Space Instrumentation for Astronomy XVI, edited by Oswald H. Siegmund, Proc. of SPIE Vol. 7435, 74350Q - (C) 2009 SPIE CCC code: $0277-786 \times / 09 / \$ 18 \cdot$ doi: $10.1117 / 12.825711$ 
Recently, ISAS has developed CdTe diode pixel detectors using aluminum as pixelated anode and Pt as the common cathode (Al-pixel/CdTe/Pt). ${ }^{6}$ Because of the Schottky diode characteristics of the Al anode contact, the low leakage current and good energy resolution have been achieved from this configuration. ${ }^{7}$

In this paper, we report the status of our development effort and the achieved performance of these novel $\mathrm{Al}$ pixel/CdTe/Pt detectors hybridized with the NuSTAR ASICs and describe results from detailed X-ray testing of the device, including spatial uniformity response. These detectors have potential application for future missions such as the International X-ray Observatory (IXO).

\section{CDTE PIXEL DETECTOR}

Figure 1 shows a picture of (a) a CdTe hybrid detector mounted on the carrier board which controls the ASIC, b) the anode side of CdTe sensor before hybridization, and c) the ASIC.

As shown in figure 1a, CdTe sensor is located at top-left corner of the carrier board. The cathode side is face up and negative high voltage is applied through the carrier board. The ASIC is sitting underneath the CdTe sensor and is wire bonded to the carrier board.

The CdTe sensor and the ASIC are flip-chip bonded with gold studs and form a hybrid detector. The distance between the detector and the ASIC is $50 \mu \mathrm{m}$. The ASIC is direct bonded to a detector with matching pixel pitch resulting in low input capacitance and very low noise operation.
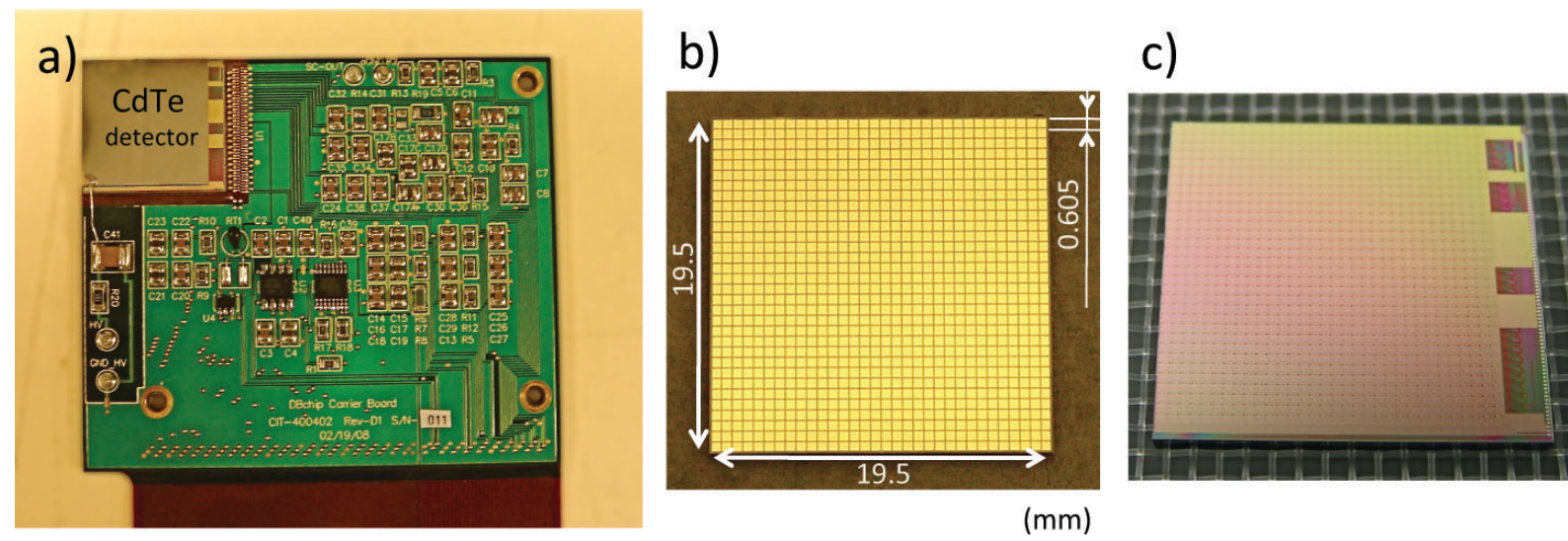

Figure 1. Photograph of the CdTe pixel detector. a) CdTe hybrid detector (CdTe+ASIC) is mounted on the carrier board. Detector is located at top-left corner. b) Anode side of CdTe sensor before hybridized to the ASIC. CdTe sensor has a size of $19.5 \times 19.5 \mathrm{~mm}^{2}$ and a thickness of $1.0 \mathrm{~mm}$. Anode pixel pitch is $605 \mu \mathrm{m}$. c) NuSTAR ASIC. The ASIC has $32 \times$ 32 readout array.

\subsection{CdTe sensor}

The CdTe sensor has a size of $19.5 \times 19.5 \mathrm{~mm}^{2}$ and a thickness of $1.0 \mathrm{~mm}$. The CdTe crystal is manufactured by ACRORAD in Japan using the Traveling Heater Method (THM). This newly developed CdTe sensor has segmented Schottky blocking contacts with a configuration of Al-pixel/CdTe/Pt. The Pt side is used as the common cathode and the $\mathrm{Al}$ side is divided into $32 \times 32=1024$ pixels. Contact are sized $545 \times 545 \mu \mathrm{m}$ with a $605 \mu \mathrm{m}$ pitch. This segmented blocking contact affords low leakage current and yet at the same time allows us to DC couple an anode contact directly to readout circuitry. ${ }^{7}$ Therefore, it is possible to fabricate electroncollecting-type diode pixel detectors that enable direct bonding to the ASIC developed for the NuSTAR space mission. 


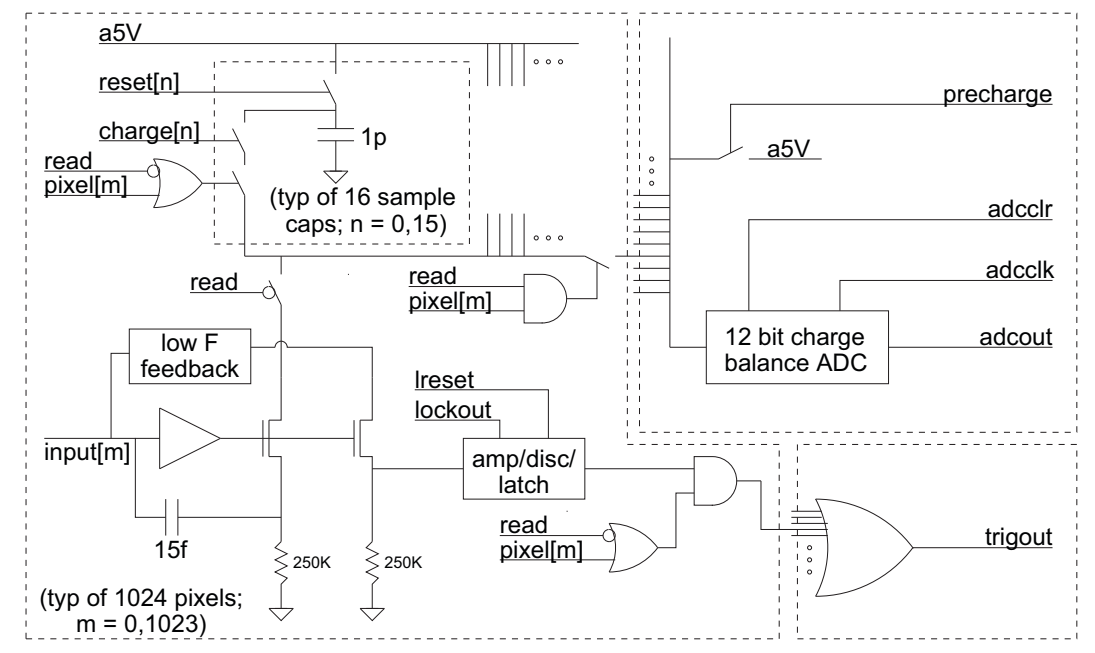

Figure 2. Schematic diagram of the ASIC readout circuitry associated with each pixel.

\subsection{ASIC}

For this study we have used the ASIC developed for NuSTAR. The ASIC contains 1024 identical readout channels in a $32 \times 32$ array with a pitch between input pads of $605 \mu \mathrm{m}$. Each channel includes a low-noise preamplifier, 16 sampling capacitors, a shaping amplifier, a discriminator and a latch. Figure 2 shows a diagram of the ASIC's readout circuitry. The output of each preamplifier presents a voltage signal to a shaping amplifier which in turn drives a discriminator input. A photon of energy above a threshold will trigger the discriminator, set a latch, and signal off-chip logic to begin an event processing cycle. This threshold is programmable from $2-40 \mathrm{keV}$. The output of each preamplifier is also presented as a current signal to a bank of 16 sampling capacitors. Analog switches route the current signal in turn to each capacitor. The process of acquiring the current-integrated preamplifier output samples proceeds continuously until an above-threshold event trigger occurs. At the time of photon detection the recent time history of the preamplifier output waveform is present on the bank of 16 capacitors. These samples record preamplifier's step-like response to the photon event. Off-chip logic circuitry then scans the chip to determine which of the 64 channels have been triggered. For the triggered channels and optionally their nearest neighbors the stored charge on each of the 16 capacitors is read out using an on-chip readout amplifier, together with analog switches that are used to route the stored charges. The readout sequence is designed to transfer the stored charges to a charge-rebalance ADC which is also included in the ASIC.

The user can select one of two methods of DC feedback for the preamplifiers, both of which allow direct detector coupling. The normal mode feedback handles leakage currents up to about 10nA, enabling operation at temperatures up to $40^{\circ} \mathrm{C}$ for typical CdZnTe detector leakage currents. The charge pump mode feedback achieves very low noise (near 200eV FWHM), and handles leakage currents up to about 300pA.

The overall gain of the readout circuits, the discrimination level, and other aspects of chip operation are programmable via the serially loaded on-chip command register. The circuit gain can be adapted over a wide range, yielding dynamic ranges suitable for the astrophysical hard X-ray telescope (2-150keV), as well as ground based radiation monitoring up to $3 \mathrm{MeV}$.

\section{DETECTOR CHARACTERIZATION}

\subsection{Leakage current}

As described on previous section, operating the ASIC with charge pump mode will achieve the best performance. However, charge pump mode has a limitation of handling detector leakage current up to about 300pA per pixel. According to the specification of our CdTe sensor provided by manufacture, the bulk leakage current reaches 

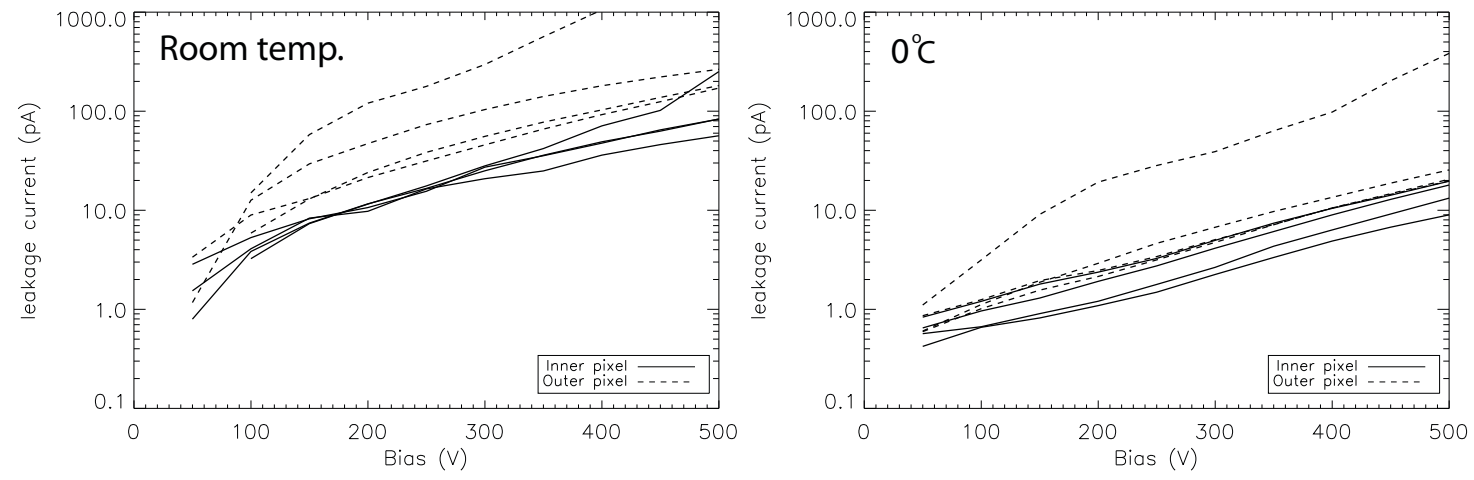

Figure 3. I-V curves of selected 8 pixels for room temperature (left) and $0^{\circ} \mathrm{C}$ degree (right). Solid and broken lines represent leakage current measured from pixels of inner region of detector and edge region respectively.

300nA around a bias voltage of $-300 \mathrm{~V}$. Since, the sensor has 1024 anode pixels, 300nA of bulk leakage current is approximately 300pA per pixel. Therefore, it is essential to cool down the CdTe to operate ASIC in charge pump mode.

Figure 3 shows the I-V curve of eight pixels (evenly spaced four inner pixels and four edge pixels) at room temperature (left) and $0^{\circ} \mathrm{C}$ degree (right) respectively. Leakage currents have been measured using the built in capability of the ASIC to probe selected pixels. Solid and broken lines represent leakage currents measured from the pixels of inner region (pixel $[11,11],[11,22],[22,11]$ and $[22,22])$ of detector and edge region ([22,0], $[0,11],[31,22]$, and [11,31]) respectively. Nominal pixel leakage currents for $-300 \mathrm{~V}$ bias voltage are $20 \mathrm{pA}$ at room temperature, and $2 \mathrm{pA}$ at $0^{\circ} \mathrm{C}$. The equivalent resistivity of the detector at room temperature is $\sim 5.4 \times$ $10^{11} \Omega \mathrm{cm}$. Note that due to the design, the CdTe sensor does not have a guard-ring, so the edge pixels have a higher leakage current.

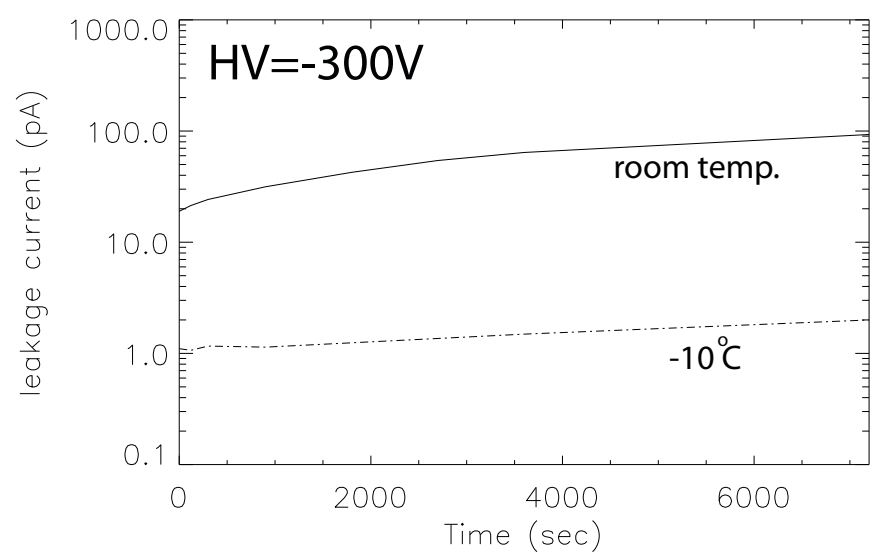

Figure 4. Leakage current vs. time since bias voltage turned on. Bias voltage is set to $-300 \mathrm{~V}$. Room temperature and $-10^{\circ} \mathrm{C}$ are shown as solid and broken lines respectively.

Leakage current stabilities have also been studied. Figure 4 shows the leakage current variation with time since turning of the high voltage. Curves in the figure represent median of leakage current for an inner $12 \times$ 12 pixel region. The leakage current shows a general trend of gradually increasing with time. We are further investigating the reason for this increase in leakage current with time; however, our results indicate that the leakage current for most of the pixel is sufficiently low to operate in charge pump mode when we cool down the detector below $0^{\circ} \mathrm{C}$. 

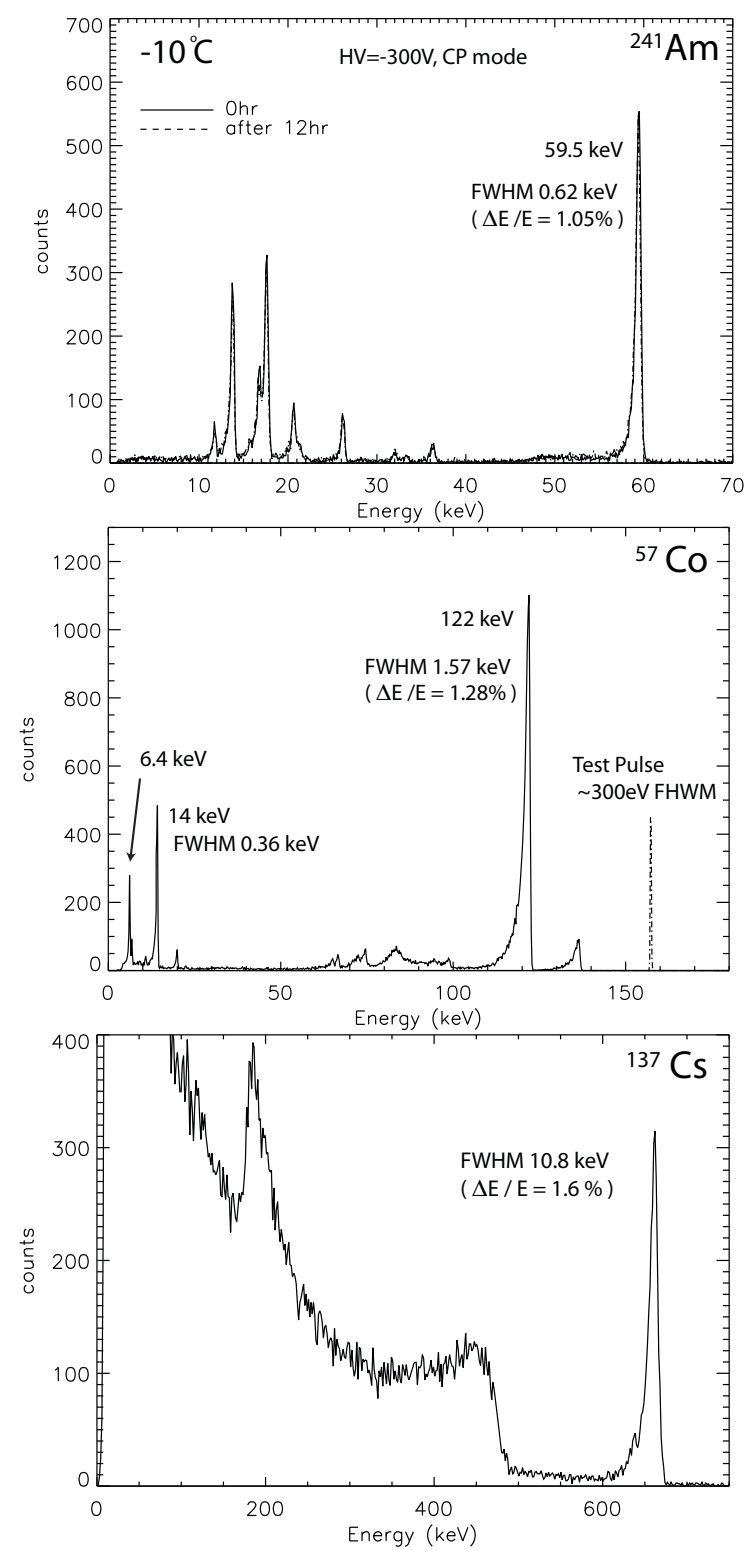

Figure $5 .{ }^{241} \mathrm{Am},{ }^{57} \mathrm{Co}$ and ${ }^{137} \mathrm{Cs}$ spectra obtained with $5 \times 5$ pixels region of the CdTe hybrid. The operating temperature was $-10^{c}$ irc $\mathrm{C}$ and the applied bias voltage was $-300 \mathrm{~V}$. Top panel shows an spectrum obtained with ${ }^{241}$ Am. The energy resolution at $59.5 \mathrm{keV}$ is $0.62 \mathrm{keV}$ FWHM and stable at least for 12 hours. Middle panel shows an spectrum obtained with ${ }^{57} \mathrm{Co}$ and test pulse. The energy resolutions for 14 and $122 \mathrm{keV}$ lines are 0.36 and $1.57 \mathrm{keV}$ FWHM respectively and test pulse FWHM is $300 \mathrm{eV}$. Bottom panel shows an spectrum obtained with ${ }^{137} \mathrm{Cs}$. The energy resolution is $10.8 \mathrm{keV}$ FWHM at $662 \mathrm{keV}$.

\subsection{Spectral resolution for X-ray events}

The X-ray spectral resolution of our detector has been measured with various radioactive sources. Figure 5 shows X-ray spectra of ${ }^{241} \mathrm{Am},{ }^{57} \mathrm{Co}$ and ${ }^{137} \mathrm{Cs}$ from top to bottom. Data are obtained by flood illumination of these radio active sources at $-10^{\circ} \mathrm{C}$ with $-300 \mathrm{~V}$ bias voltage applied. Spectra are produced by adding pixels from a $5 \times 5$ region of $\mathrm{CdTe}$ detector. These spectra include both single-pixel and multiple-pixel triggered events. 
Appropriate corrections have been applied to all spectra including pixel by pixel gain calibrations. ${ }^{8}$

The top panel shows a ${ }^{241} \mathrm{Am}$ spectrum. The energy resolution is $0.62 \mathrm{keV} \mathrm{FWHM} \mathrm{at} 59.5 \mathrm{keV}$. We collected the data promptly after turning on the bias voltage and after 12 hours to study effect of polarization. The two spectra are shown in top panel as solid and dashed lines. The two spectra are almost indistinguishable from each other indicating there is no spectrum degradation for at least 12 hours.

The middle panel shows a ${ }^{57} \mathrm{Co}$ spectrum. The energy resolutions for the 14 and $122 \mathrm{keV}$ lines are 0.36 and 1.57keV FWHM respectively. Note that the Fe-K $(6.4 \mathrm{keV})$ line is clearly represented which indicates low noise and good energy resolution. The test pulse is shown as a dashed line. The energy resolution of test pulse is $300 \mathrm{eV}$ FWHM which is typical number for all three measurements.

The bottom panel shows a ${ }^{137} \mathrm{Cs}$ spectrum. The energy resolution is $10.8 \mathrm{keV}$ FWHM at $662 \mathrm{keV}$. In order to operate over the wider energy band to obtain the ${ }^{137} \mathrm{Cs}$ spectrum, we used lower gain and higher sampling frequency. Note that due to the CdTe detector thickness $(1 \mathrm{~mm})$ and radioactive source availability, we need to irradiate ${ }^{137} \mathrm{Cs}$ more than 24 hours to obtain the results which indicates good stability of our detector. In order to study the high energy response, we need to use a thicker detector for actual applications.

\subsection{Polarization}

Similar to the In/CdTe/Pt Schottky barrier type detector, polarization effects are also seen with Al-pixel/CdTe/Pt type detector at the room temperature. Figure 6 shows an ${ }^{241} \mathrm{Am}$ source spectrum measured for 4 hours. ASIC has operated in normal mode to handle the high leakage current at the room temperature. Due to this operation mode, electronic noise is slightly higher than the charge pump mode (see figure 5): $1.4 \mathrm{keV} \mathrm{FWHM} \mathrm{at} 59.5 \mathrm{keV}$. Besides this initial energy resolution difference, room temperature spectral resolution starts to degrade shortly after the HV is turned on. Figure 6 shows spectrum after 1 hour (dash line) and 4 hour (dotted line) HV turned on, which clearly show a degradation of the spectrum. Note that after 4 hour of measurement, the HV was turned off for a second to confirm that spectral shape recovers to the initial spectral response.

However, with the detector cooled down below $0^{\circ} \mathrm{C}$, there is no serious polarization effect observed, as seen from Figure 5a.

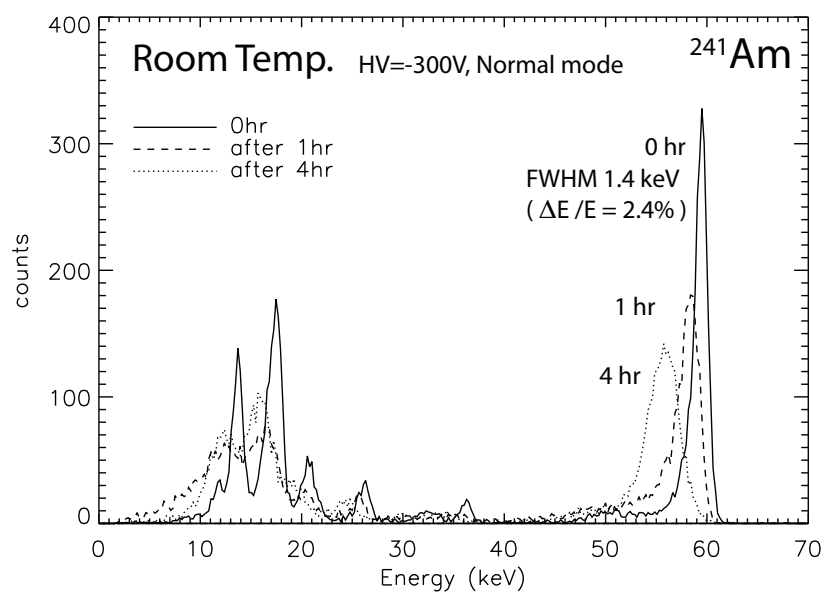

Figure 6. ${ }^{241} \mathrm{Am}$ X-ray spectra obtained with CdTe hybrid detector with Normal mode operation. Bias voltage applied $-300 \mathrm{~V}$ at room temperature. Energy resolution first seen as $1.4 \mathrm{keV}$ at $59.5 \mathrm{keV}$ was soon degradate shown as 1 hour and 4 hour due to the polarization.

\subsubsection{Uniformity of the detector and Imaging}

Next, we have studied the count rate uniformity distribution over the hybrid detector, since it reflects the variations of the detection efficiency in the detector. Figure 7 shows the event count distribution obtained with ${ }^{241} \mathrm{Am}$ source flood illumination in a gray scale map on the left and the distribution histogram on the right. 

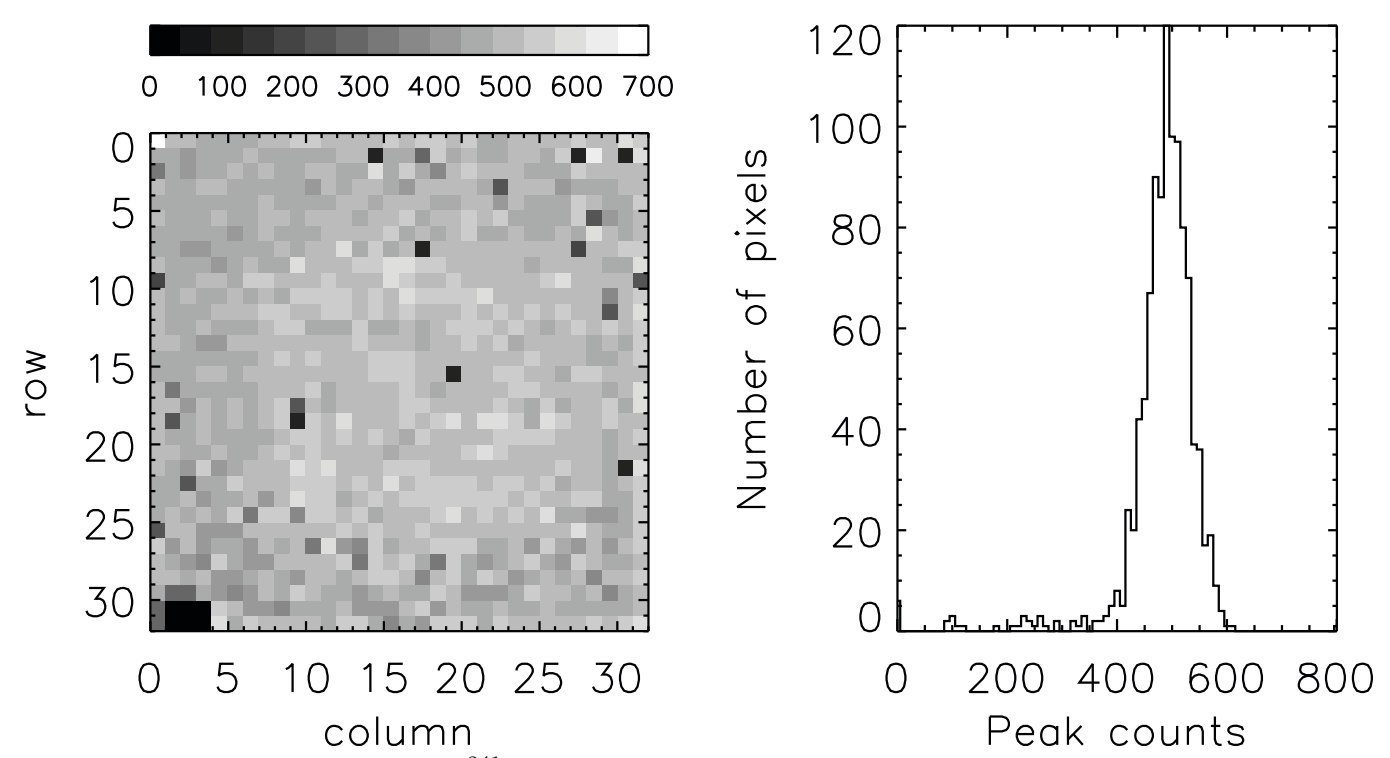

Figure 7. Counts rate map obtained with ${ }^{241} \mathrm{Am}$ flood illumination. An uniformity distribution over the hybrid detector in a gray scale map in the left and in the histogram in the right.

Pixels in the lower left corner were turned off during this test. There are a few pixels with significantly lower counts than mean distribution. Considering that most of those pixels' test pulses were working properly, some kind of disconnection between anode and ASIC may have happened. In contrast, we see far fewer potentially disconnected pixels with CdZnTe detectors.
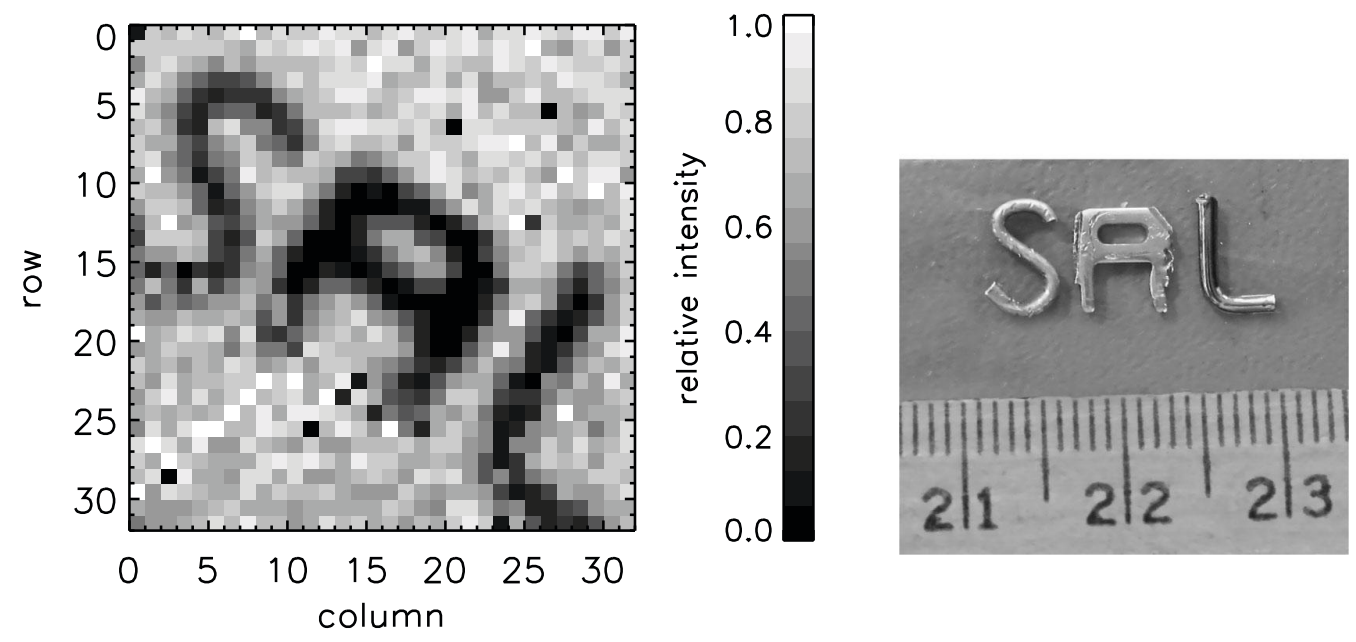

Figure 8. Shadow image obtained with CdTe hybrid and a photograph of the mask. The image obtained with a flood illumination of ${ }^{241} \mathrm{Am}$ on top of the "SR" mask.

Excluding outlier low count rate pixels, the standard deviation is $7.9 \%$, which is larger than the Poisson distribution of $4.5 \%$. This variation could be explained by the variations of pixel effective area but we need farther investigations of this non-uniformity. 
To estimate the imaging capability of this CdTe detector, we prepared a "SRL" (Space Radiation Laboratory) mask and took flood illumination data using ${ }^{241} \mathrm{Am}$ source with mask in between source and the detector. We also took a flat field image using ${ }^{241} \mathrm{Am}$ flood data without the mask. The image shown in left panel of figure 8 was obtained by dividing the masked image with flat field image. The color bar shows relative intensity in the image. The right panel shows actual mask used to generate this image. The image clearly shows pixel level resolution for this CdTe detector which in turn suggests that the observed non-uniformity in counts is stable with time.

\section{SUMMARY}

We have successfully developed a hybrid detector using anode pixelated Al-pixel/CdTe/Pt detector and the NuSTAR ASIC. We achieved very good energy resolution over a wide energy range for this hybrid: $0.36 \mathrm{keV}$ FWHM @ 14keV, 0.62keV FWHM @ 60keV, 1.57keV FWHM @ 122keV and 10.8keV FWHM @ 662keV.

The nominal per pixel leakage currents of this detector for $-300 \mathrm{~V}$ bias voltage are $20 \mathrm{pA}$ at room temperature and $2 \mathrm{pA}$ at $0^{\circ} \mathrm{C}$. The equivalent resistivity of the detector at room temperature is $\sim 5.4 \times 10^{11} \Omega \mathrm{cm}$. We observed a gradual increase in leakage current with time; this issue is still under investigation.

Polarization has been observed at room temperature for the CdTe detectors. The X-ray spectrum degraded noticeably in less than an hour due to polarization. However, if the detector is cooled down below $0^{\circ} \mathrm{C}$, spectral degradation is significantly suppressed and there is no serious polarization effect observed for at least 12 hours.

We observed some non-uniformity in the counts distribution. We speculate that handling procedures or the bonding process may have cause the observed non-uniformity. However further investigating is required on this issue. Pixel-level resolution on the mask image indicates that the non-uniformity of detection efficiency is stable in time.

\section{REFERENCES}

[1] Hubert Chen, C., Cook, W., Harrison, F., and Lin, J., "Spatial characterization of a large-format, fine-pitch CdZnTe pixel detector for the HEFT balloon-borne experiment," 3346-50 Vol.5, IEEE, Piscataway, NJ, USA (2004 2004).

[2] Cook, W., Boggs, S., Bolotnikov, A., Burnham, J., Fitzsimmons, M., Harrison, F., Kecman, B., Matthews, B., and Schindler, S., "High resolution CdZnTe pixel detectors with VLSI readout," IEEE TRANSACTIONS ON NUCLEAR SCIENCE 47, 1454-1457 (AUG 2000).

[3] Harrison, F. A., Boggs, S. E., Bolotnikov, A. E., Christensen, F. E., Cook, W. R., Craig, W. W., Hailey, C. J., Jimenez-Garate, M. A., Mao, P. H., Schindler, S. M., and Windt, D. L., "Development of the HighEnergy Focusing Telescope (HEFT) balloon experiment," Society of Photo-Optical Instrumentation Engineers (SPIE) Conference Series 4012, 693-699 (July 2000).

[4] Harrison, F. A., Christensen, F. E., Craig, W., Hailey, C., Baumgartner, W., Chen, C. M. H., Chonko, J., Cook, W. R., Koglin, J., Madsen, K.-K., Pivavoroff, M., Boggs, S., and Smith, D., "Development of the HEFT and NuSTAR focusing telescopes," Experimental Astronomy 20, 131-137 (Dec. 2005).

[5] Oonuki, K., Inoue, H., Nakazawa, K., Mitani, T., Tanaka, T., Takahashi, T., Chen, C. M. H., Cook, W. R., and Harrison, F. A., "Development of uniform CdTe pixel detectors based on Caltech ASIC," 5501, 218-228 (Sept. 2004).

[6] Toyama, H., Nishihira, A., Yamazato, M., Higa, A., Maehama, T., Ohno, R., and Toguchi, M., "Formation of aluminum Schottky contact on plasma-treated cadmium telluride surface," JAPANESE JOURNAL OF APPLIED PHYSICS PART 1-REGULAR PAPERS SHORT NOTES 43, 6371-6375 (Sept. 2004).

[7] Watanabe, S., Ishikawa, S.-n., Takeda, S., Odaka, H., Tanaka, T., Takahashi, T., Nakazawa, K., Yamazato, M., Higa, A., and Kaneku, S., "New CdTe pixel gamma-ray detector with pixelated Al schottky anodes," JAPANESE JOURNAL OF APPLIED PHYSICS PART 1-REGULAR PAPERS BRIEF COMMUNICATIONS \& REVIEW PAPERS 46, 6043-6045 (SEP 2007).

[8] Rana, V. R., Cook, W. R., Harrison, F. A., Mao, P. H., and Miyasaka, H., "Performance of the NuSTAR focal plane detectors," SPIE Conference Series To appear (2009). 